\title{
X-Ray Digital Tomosynthesis Imaging: An Appropriate Reconstruction Algorithm for Arthroplasty
}

\author{
Tsutomu Gomi, Hiroshi Hirano and \\ Masahiro Nakajima \\ Additional information is available at the end of the chapter \\ http://dx.doi.org/10.5772/53248
}

\section{Introduction}

Digital tomosynthesis is a limited-angle method for image reconstruction. In this technique, a projection dataset of an object acquired at regular intervals during a single acquisition pass is used to reconstruct planar sections post priori. Tomosynthetic slices exhibit high resolution in planes that are parallel to the detector plane. Digital tomosynthesis enhances the existing advantages of conventional tomography, including low radiation dose, short examination time, and easy, low-cost availability of longitudinal tomographs, which do not include the partial volume effect. Furthermore, digital tomosynthesis provides the additional benefits of digital imaging (Ziedses et al 1971, Miller et al 1971, Grant et al 1972, Baily et al 1973, Kruger et al 1983, Sone et al 1991, Sone et al 1995) as well as the tomographic benefits of computed tomography $(\mathrm{CT})$ at a decreased radiation dose and cost in an approach that is easily implemented in conjunction with chest radiography. This technique was developed by improving the old technique of geometric tomography, which is unpopular for chest imaging because of positioning difficulties, high radiation dose, and residual blur due to out-of-plane structures. Digital tomosynthesis overcomes these difficulties by enabling the reconstruction of numerous image slices from the data acquired from a single low-dose image. Digital tomosynthesis images are invariably affected by blurring because of objects lying outside the plane of interest and those superimposed on the focused image of the fulcrum plane by the limited acquisition angle. This results in poor object detectability in the in-focus plane. This technique has been investigated in angiography and the imaging of chest, hand joints, lungs, teeth and breasts (Stiel et al 1993, Duryea et al 2003, Sone et al 1995, Niklason et al 1997, Dobbins et al 2003). 
Imaging by X-ray CT has improved over the past three decades and is now a powerful tool in medical diagnostics. It has become an essential, non-invasive imaging technique since the advent of spiral CT imaging in the 1990s, which led to shorter scan times and improved three-dimensional (3D) spatial resolution. CT provides a high resolution in the tomographic plane but limited resolution in the axial direction. However, the quality of images generated by a CT scanner can still be reduced by the presence of metal objects in the field-of-view. Imaging patients with metal implants such as marker pins, dental fillings or hip prostheses is susceptible to artifacts, generally in the form of bright and dark streaks, cupping, capping and so on. This artifact susceptibility is mostly due to quantum noise, scattered radiation and beam hardening (Hsieh 1995). Metal artifacts influence the image quality by reducing the contrast and obscuring details, thus hindering the ability to detect structures of interest and possibly leading to misdiagnosis. In addition, CT values are reduced, which can lead to errors while using these data e.g. for attenuation correction in positrin emission tomography (PET)/CT imaging (Kamel et al 2003). The metallic components of arthroplasty devices are high-contrast objects that generate artifacts when imaged using CT scans. These artifacts can make it extremely difficult or impossible to interpret images obtained by these devices. The presence of artifacts, along with the partial volume effect, severely limits the potential for the objective quantification of total joint replacement with CT.

Methods for reducing metal artifacts aim to improve the quality of images, affected by them. Recently, modified- iterative (Wang et al 1996, 1999, 2000, Man et al 2000) or wavelet-reconstruction techniques have produced promising results. However, these methods cannot be combined with the fast, robust filtered backprojection (FBP) algorithm, which is the standard reconstruction technique (Robertson et al 1997) implemented in modern CT scanners.

Digital tomosynthesis using the FBP algorithm shows satisfactory overall performance, but its effectiveness depends strongly on the region of the image. This type of digital tomosynthesis gives good results independent of the type of the metal present in the patient and effectively removes noise artifacts, especially at greater distances from the metal objects (Gomi et al 2008). In addition, flexibility in choosing digital tomosynthesis imaging parameters on the basis of the desired final images and generation of high-quality images may be beneficial.

In this study, we focus on the potential application of digital tomosynthesis using a different algorithm for an enhanced performance, which is used for imaging hip prosthesis phantoms (titanium) and human hip prostheses. The present study was performed to evaluate the clinical application of digital tomosynthesis in imaging these objects using the relatively commercial tomosynthesis method. Digital tomosynthesis was compared to the use of conventional reconstruction for tomosynthesis (FBP), metal-artifact-reduction processing reconstruction for tomosynthesis, iterative reconstruction for tomosynthesis [simultaneous iterative reconstruction technique; SIRT (Gordon et al 1970)], adaptive statistical iterative reconstruction $\mathrm{CT}$ and non-metal-artifact-reduction processing CT (conventional FBP reconstruction) scans of a hip prosthesis phantom and human hip prosthetic case. 


\section{Image reconstruction}

\subsection{Tomosynthesis}

Existing tomosynthesis algorithms can be divided into three categories: (1) backprojection algorithms, (2) FBP algorithms, and (3) iterative algorithms. The backprojection algorithm is referred to as a shift-and-add (SAA) process, in which the projection images obtained at different angles are electronically shifted and added to generate an image plane, focused at a certain depth below the surface. The projection shift is adjusted such that the visibility of features in the selected plane is enhanced, whereas that in other planes is blurred. By using a digital detector, the image planes at all depths can be retrospectively reconstructed from one set of projections. The SAA algorithm is valid only if the motion of the X-ray focal spot is parallel to the detector (Gomi et al 2012).

In FBP algorithms, which are widely used in $\mathrm{CT}$, many projections acquired at greater than $360^{\circ}$ are used to reconstruct cross-sectional images. The number of projections typically ranges between a few hundred and approximately one thousand. The Fourier central slice theorem is fundamental to the FBP theory. In two-dimensional (2D) CT imaging, projecting an object corresponds to sampling it perpendicular to the X-ray beam in Fourier space (Kak et al 1988). For many projections, information of the object is well sampled, and the object can be restored by combining the information from all the projections. In 3D cone-beam imaging, the information of the object in Fourier space is related to the Radon transform of the object. The relationship between the Radon transform and conebeam projections has been studied properly, and solutions to the cone-beam reconstruction have been provided (Smith 1985). The FBP algorithm generally provides highly precise 3D reconstruction images when an exact-type algorithm is employed (Feldkamp et al 1984). Therefore, this method has been adopted for the image reconstruction of 3D tomography and multi-detector cone-beam CT.

An iterative algorithm performs reconstruction recursively (Ruttimann et al 1984, Bleuet et al 2002), unlike the one-step operation in backprojection and FBP algorithms. During the iterative reconstruction, a 3-D object model is repeatedly updated until it converges to the solution, which optimizes an objective function. The objective function defines the criteria of the reconstruction solution. The objective function in SIRT are applied iteratively so that the projections of the reconstructed volume, computed from an image-formation model, resemble the experimental projections. A linear-projection model is a first-order approximation of the nonlinear image-formation process, occurring in tomosynthesis. Furthermore, although the noise is not white, the SIRT formula for white noise produces good estimates of the underlying structures.

Metal artifacts influence the image quality by reducing the contrast and obscuring the detail, thereby impairing the ability to detect structures of interest and making diagnosis impossible. Artifacts due to high-attenuation features in hip prostheses are observed in digital-tomosynthesis reconstruction because of the small number of projections and narrow angular range, typically employed in tomosynthesis imaging developed artifact-reduction methods 
on the basis of a modified Shepp-Logan reconstruction filter kernel by considering the additional weight of the direct current components in the frequency domain (Gomi et al 2009). Processing increases the ratio of low-frequency components in an image (Fig. 1). Artifact was reduced using basic and FBP algorithms. It provides a filtering method that can be used in combination with the backprojection algorithm to yield sliced images with the desired properties via tomosynthesis.

The tomosynthesis system (SonialVision Safire II, Shimadzu Co., Kyoto, Japan) comprised an X-ray tube with a $0.4-\mathrm{mm}$ focal spot and a $362.88 \times 362.88-\mathrm{mm}$ digital flat-panel detector composed of amorphous selenium. Each detector element was $150 \times 150 \mu \mathrm{m}$ in size. Tomography was performed linearly with a total acquisition time of $6.4 \mathrm{~s}\{80 \mathrm{kVp}, 250 \mathrm{~mA}, 20 \mathrm{~ms} /$ view, effective dose: $1.33 \mathrm{mSv}$ [International Commission on Radiological Protection (ICRP) 60], $0.69 \mathrm{mSv}$ (ICRP 103)\} and an acquisition angle of 40 degree. Projection images were sampled during a single tomographic pass (74 projections) using a matrix size of $1440 \times 1440$ with 12 bits per image and were used to reconstruct tomograms of a desired height. The reconstructed images $(0.272 \mathrm{~mm} /$ pixel $)$ were obtained with a 4 -mm slice thickness at $1-\mathrm{mm}$ reconstruction intervals. An antiscatter grid was used (focused type, grid ratio 12:1). The distance from the source to the isocentre was $980 \mathrm{~mm}$ and that from the isocentre to the detector was $1100 \mathrm{~mm}$ (3.0-mm aluminium equivalent filtration). The tomosynthesis images were reconstructed using FBP with the conventional Shepp-Logan filter kernel.

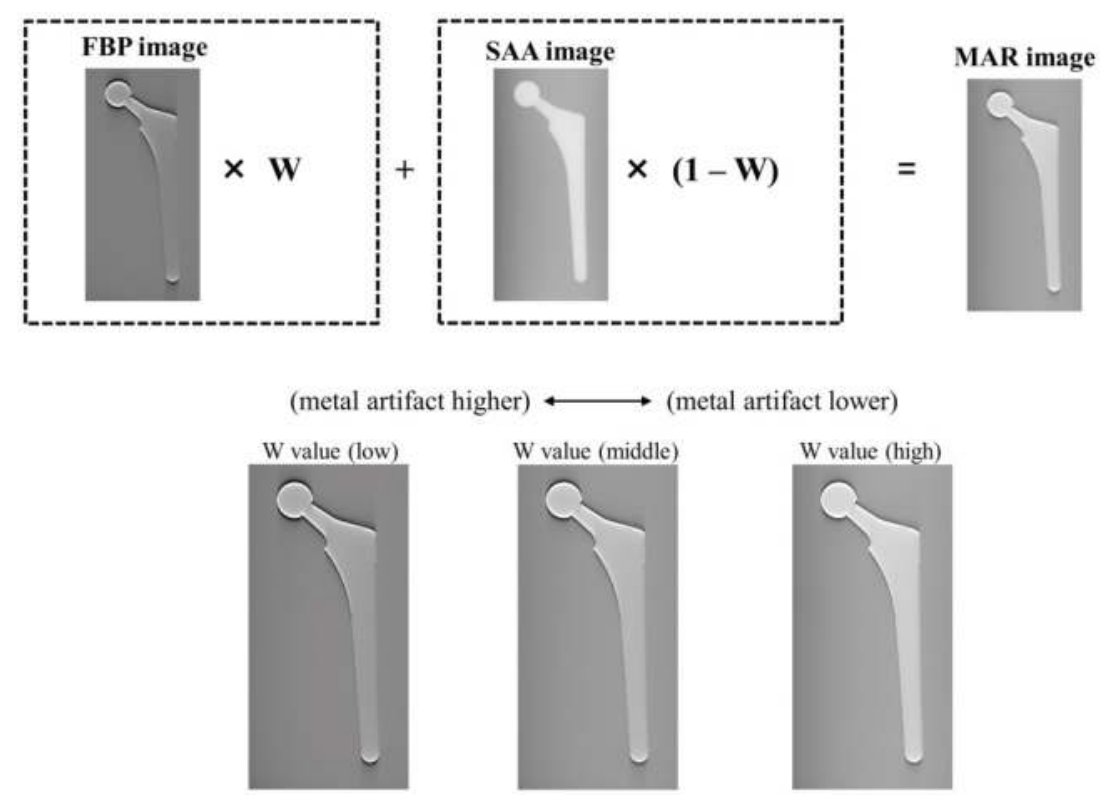

Figure 1. Concept of the metal-artifact-reduction processing method for tomosynthesis. The image is weighted by multiplying the different weight coefficients and adding them to the shift-added image and the conventional FBP image. 


\subsection{CT}

The presence of metal artifacts has been a major problem in X-ray CT. Metal parts in the field-of-view attenuate most of the $\mathrm{X}$-ray photons and generate dark and bright streaks after FBP, which is currently the selected reconstruction method for CT. These artifacts severely degrade the image quality, particularly near metal surfaces. Metal-artifact reduction has important applications in orthopaedic, oncologic, and dental imaging.

Iterative reconstruction algorithms, with and without the incorporation of a priori information, have been used to reconstruct incomplete projections. Although previous results using iterative reconstruction were unsatisfactory, a recently developed iterative-deblurring method has produced an image reconstruction of the incomplete data with few artifacts. Iterative reconstruction, which has recently become available on commercial CT scanners, enables metal-artifact-noise reduction without trade-off in spatial resolution (Main et al 2010). However, iterative reconstruction has unfavourable effects. Its use alters the texture of the image noise and can yield an unusually homogenous image. This may not be immediately appealing to most radiologists, who are usually accustomed to FBP images (Hara et al 2009). Moreover, an excessive degree of iterative reconstruction may obscure fine and subtle findings (Yanagawa et al 2010).

In computation with iterative reconstruction, the image has an initial condition of values, which are iteratively optimized according to the rules of the model. The FBP image is used for the initial condition in adaptive statistical iterative reconstruction (GE Healthcare Corp.; the initial value of each pixel) for the following reasons: it is presumably close to the final optimized solution (lessening the need for iterations), it is a valid indicator of specific-slice image noise and it can be obtained rapidly. For modelling and using iterative reconstruction, minimum convergence is achievable with adaptive statistical iterative reconstruction. However, a fully converged, $100 \%$ adaptive statistical iterative reconstruction image, has a noise-free appearance with an unusually homogeneous attenuation. Because some noise is inherent in CT, the use of $100 \%$ adaptive statistical iterative reconstruction may not be immediately appealing to most of the radiologists. However, blended images containing a linear mixture of the original FBP and this reconstruction can exhibit markedly decreased noise while retaining a more typical CT appearance. This blended image can be adjusted from $1 \%$ to $100 \%$ in adaptive statistical iterative reconstruction. The $40 \%$ level was chosen because $40 \%$ adaptive statistical iterative reconstruction should produce a diagnostically acceptable image with less noise than a full-dose FBP image. We selected the blending ratios of $20 \%$, $40 \%$, and $60 \%$ according to the results of a previous study (Hara et al 2009). In the conventional FBP reconstruction, standard reconstruction kernels were used.

CT scan was performed on a multi-slice CT scanner (64-slice Discovery CT 750HD scanner; GE Healthcare Corp., Milwaukee, WI) with $120 \mathrm{kVp}, 150 \mathrm{~mA}, 0.625 \mathrm{~mm} \times 64$ collimation, and a 1-s gantry rotation time at a beam pitch of 0.984 [effective dose, $5.4 \mathrm{mSv}$ (ICRP 60), 4.1 mSv (ICRP 103)]. The clinical task was to assess the hip prostheses. A 4-mm thick slice is generally used in clinical practice. In this study, we applied the slice thickness used during the screenings; therefore, the axial reconstructed images were obtained with a 4-mm slice thickness at $1-\mathrm{mm}$ reconstruction intervals $(512 \times 512$ pixels and $140-\mathrm{mm}$ field-of-view). 


\section{Evaluation}

In the study, the artifact-reduction performance was evaluated using the intensity profile, artifact spread function (ASF) and root-mean-square error (RMSE). The intensity profiles were compared using different reconstruction methods in the in-focus plane. Wu et al. proposed an ASF metric to quantify the artifacts observed in planes outside the focus image plane (Wu et al 2003). These artifacts are generated from real features located in the focus image plane and resemble the real feature. The artifacts exhibited in the image plane are defined by the ASF as $\frac{N_{\text {artifact }}(z)-N_{B G}(z)}{N_{\text {artifact }}\left(z_{0}\right)-N_{B G}\left(z_{0}\right)}$, where $z$ to the base of 0 is the location of the infocus plane of the real feature and $z$ is the location of the off-focus plane. $N$ to the base of artifact $(z 0)(z)$ and $N B G(z 0)$ are the average pixel intensities of the feature and the image background in the in-focus plane, respectively. Nartifact $(z)$ and $N B G(z)$ are the average pixel intensities of the artifact and the image background in the off-focus plane, respectively. Another important metric to be considered is RMSE, which can be computed by obtaining the root of the summation of the square of the standard deviation and the square of the bias. The errors in the image plane are defined in terms of RMSE as $R M S E=\sqrt{\sum_{i=1}^{n}\left(X-x_{i}\right)^{2} / n}$, where $X$ is the observed image, $x_{i}$ is the referenced image, and $n$ is the number of compounds in the analysed set.

\section{Results}

\subsection{Hip prosthesis phantoms (titanium)}

A comparison of the intensity profiles and RMSEs of the tomosynthesis and CT images revealed that tomosynthesis (metal-artifact-reduction processing and the iterative algorithm) decreased the number of metal and beam hardening artifacts in the reconstructed images. Furthermore, this technique yielded a higher contrast detectability than the existing FBP algorithm. In the reconstructed images obtained from metal-artifact-reduction processing, the quantum noise structure decreased, and the noise structure was slightly smoother (Figs. 2-6).

The chart in Fig. 7 shows the ASF results for the prosthetic case. This chart shows ASF versus the distance from the in-focus slice in millimetres. There were nine reconstructed slices. The chart demonstrates that tomosynthesis with metal-artifact-reduction processing ( $W=$ high) removes the highest number of metal artifacts. Examining Fig. 7 through the entire thickness of the specimen shows that the order of ASF performance of the algorithm is as follows: (1) tomosynthesis (metal-artifact-reduction processing with FBP, $W=$ high); (2) CT (20\%, 40\%, and 60\% adaptive statistical iterative reconstruction and conventional FBP); (3) tomosynthesis (iterative algorithm, 100 iterations); and (4) tomosynthesis (conventional FBP). 


\subsection{Human hip prostheses}

To demonstrate the potential benefits of digital tomosynthesis compared with CT in imaging hip prostheses, we used one clinical case, a 52-year-old female with total hip arthroplasty. The use of digital tomosynthesis improved the visualisation of the underlying tissue detail by blurring the overlying structures. CT provided information [multiplanar reformation (MPR) of images] on the hip prostheses, as shown in Fig. 8. MPR of CT images suffered from string artifacts in all regions. In addition, due to strong beam hardening and scattering, the femur region was poorly displayed. The artifacts in CT images, produced by FBP, were realistic and resembled actual patient images. The more metal was present in the field-ofview (metal-backed and bilateral prostheses), the more metal artifacts were produced. Reconstruction of the incomplete projection data by using iterative deblurring produced an essentially metal-artifact-free image for soft tissues and outperformed the FBP methods. The hip prostheses present on the digital tomosynthesis images could be removed effectively by blurring in the 74-projection digital tomosynthesis image. This allowed better visualisation of the tissue detail directly below the hip prostheses structures.
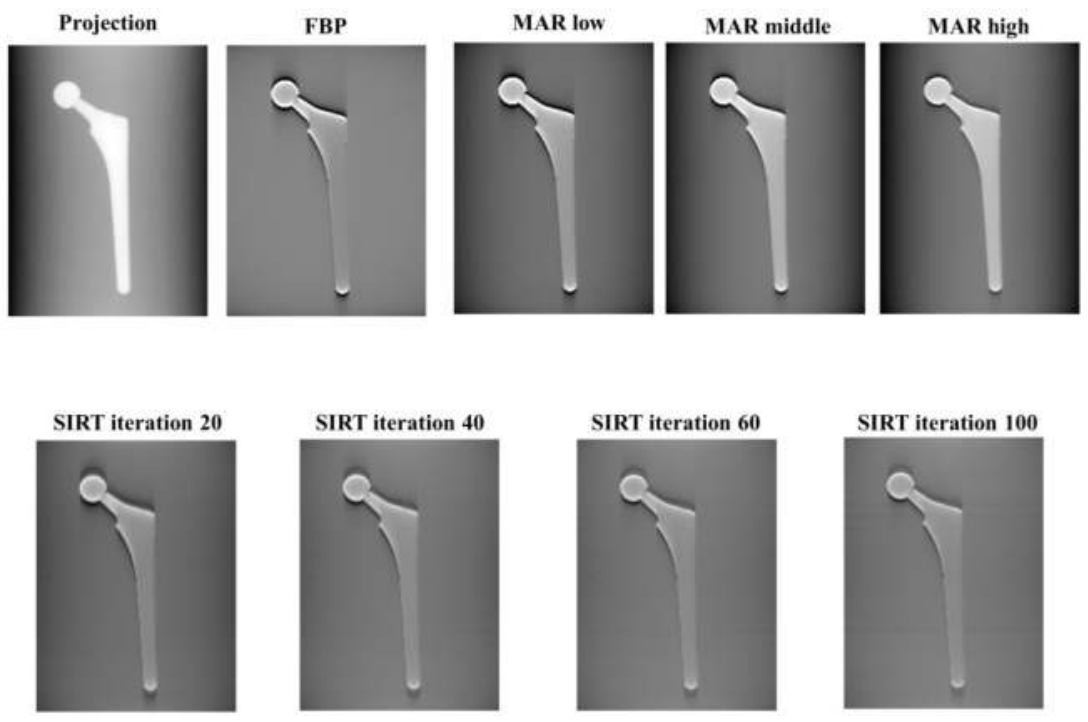

Figure 2. Comparison between tomosynthesis images and those obtained by metal-artifact-reduction processing, conventional FBP and SIRT imaging algorithms in the in-focus plane. (Reference is projection image.) 

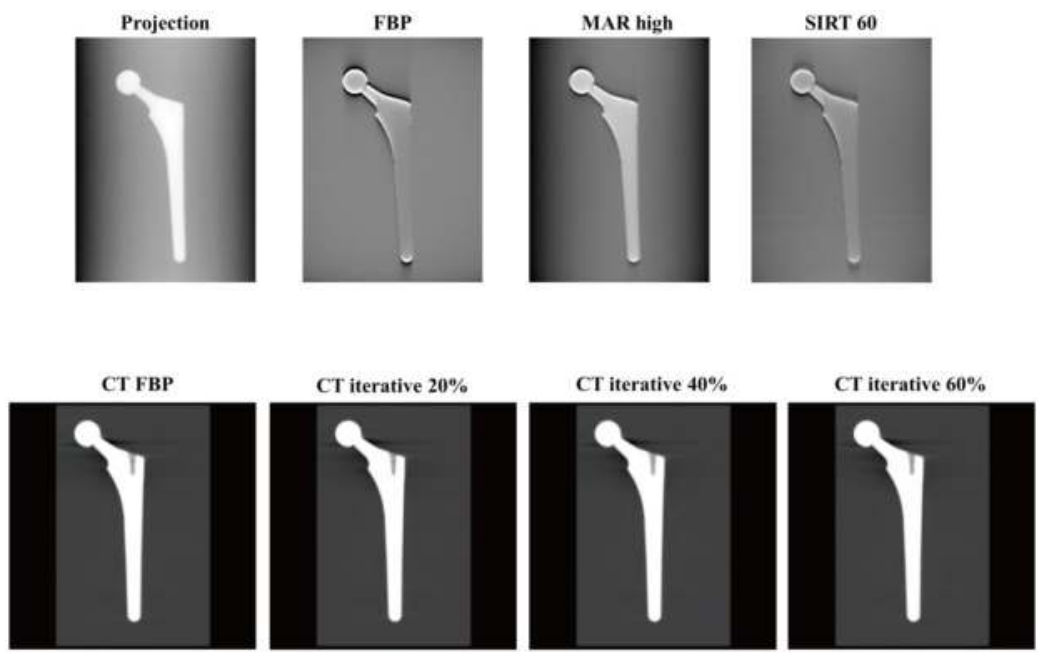

Figure 3. Comparison between excellent tomosynthesis images and those obtained by metal-artifact-reduction processing ( $W=$ high), conventional FBP, SIRT imaging algorithms (60 iterations) in the in-focus plane and CT images (conventional FBP and iterative reconstruction). Metal-artifact-reduction processing provided a better visualisation of the hip prosthesis phantom by eliminating, blurring and reducing the artifacts, above, and the visualized planes, below.

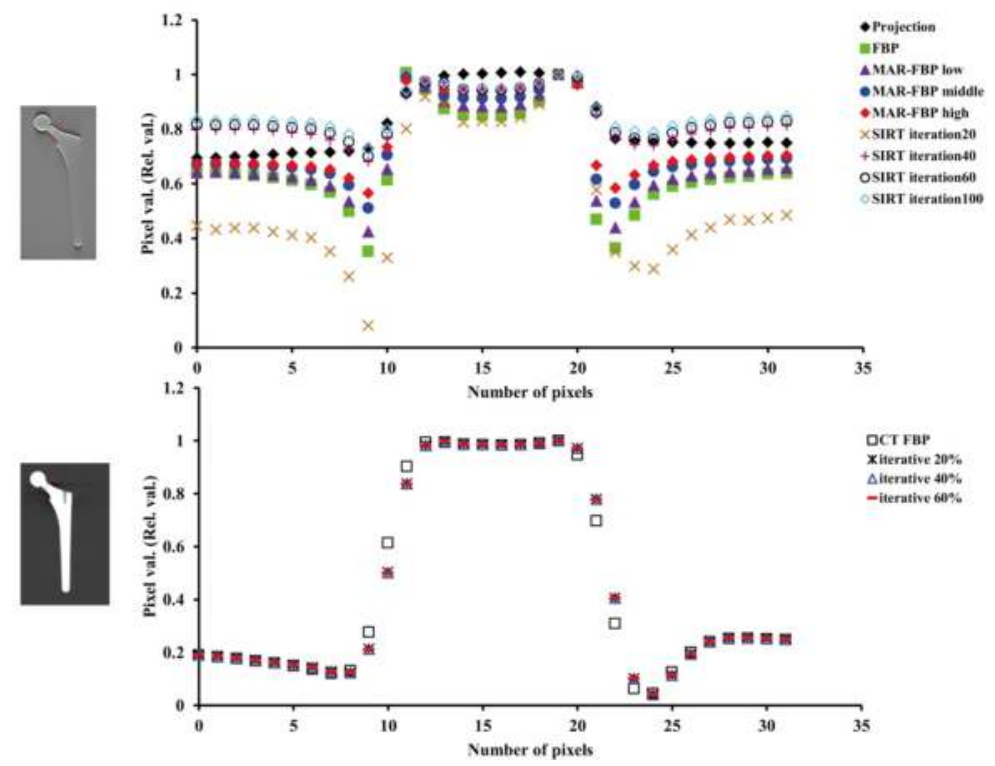

Figure 4. Comparison between intensity profiles using tomosynthesis and CT in the in-focus plane. Artifacts (part of undershooting) are reduced by metal-artifact-reduction processing and SIRT technique for tomosynthesis. 

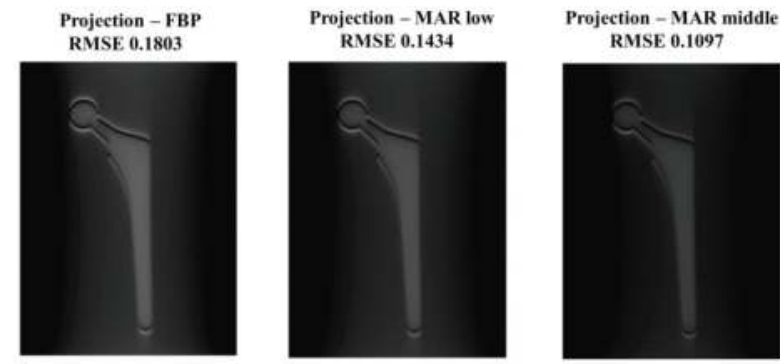

Projection - MAR high RMSE 0.0756

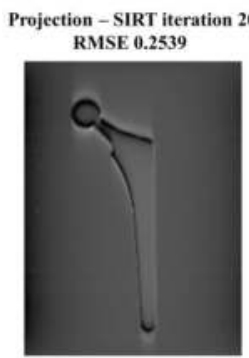

Projection - SIRT iteration 40 RMSE 0.2091

Projection -SIRT iteration 60 RMSE 0.2170
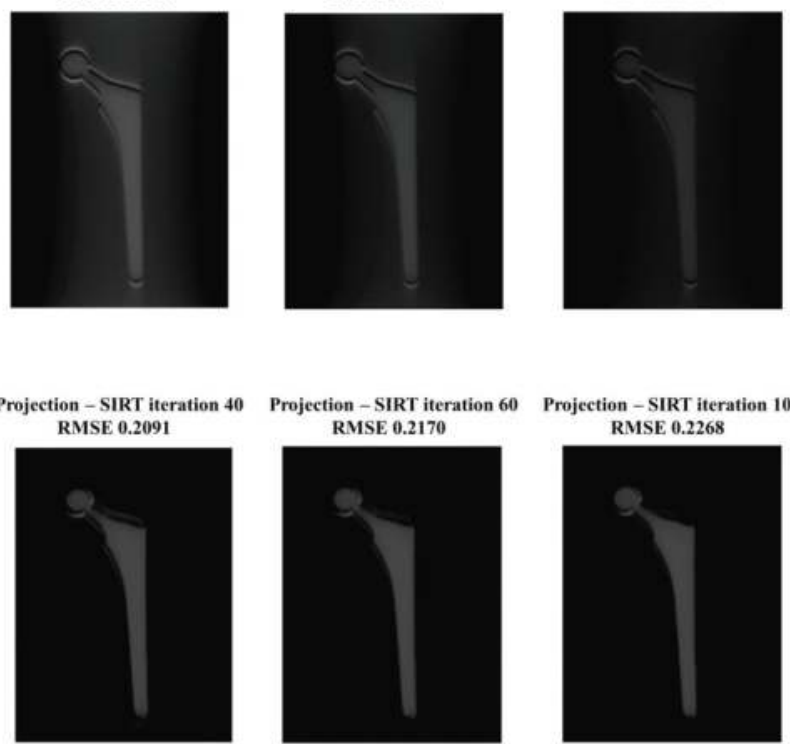

Projection - SIRT iteration 100 RMSE 0.2268

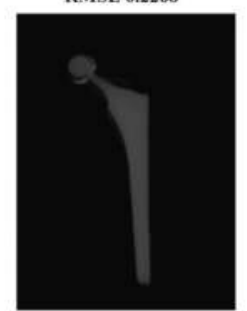

Figure 5. Comparison between tomosynthesis error images and RMSE of the images obtained by metal-artifact-reduction processing ( $W=$ low, medium and high), conventional FBP and SIRT imaging algorithms in the in-focus plane. (Reference is the projected image.)
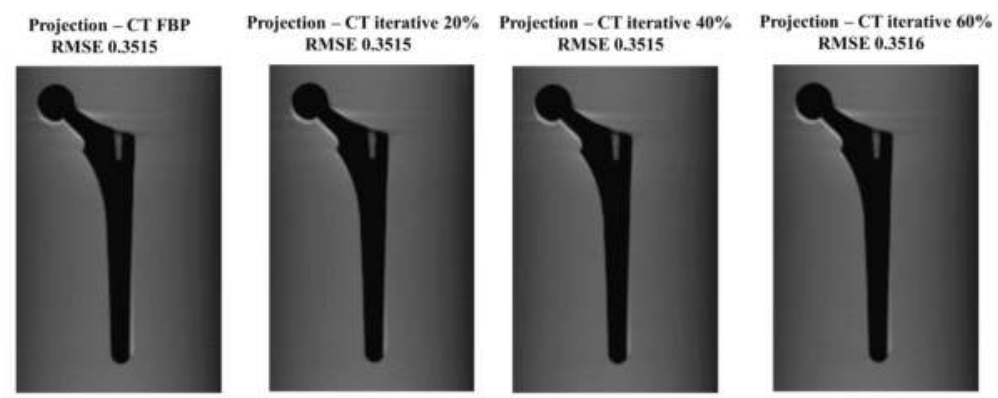

Figure 6. Comparison between CT error images and RMSE of images obtained from conventional FBP and iterative reconstruction algorithms in the in-focus plane. (Reference is the projected image.) 


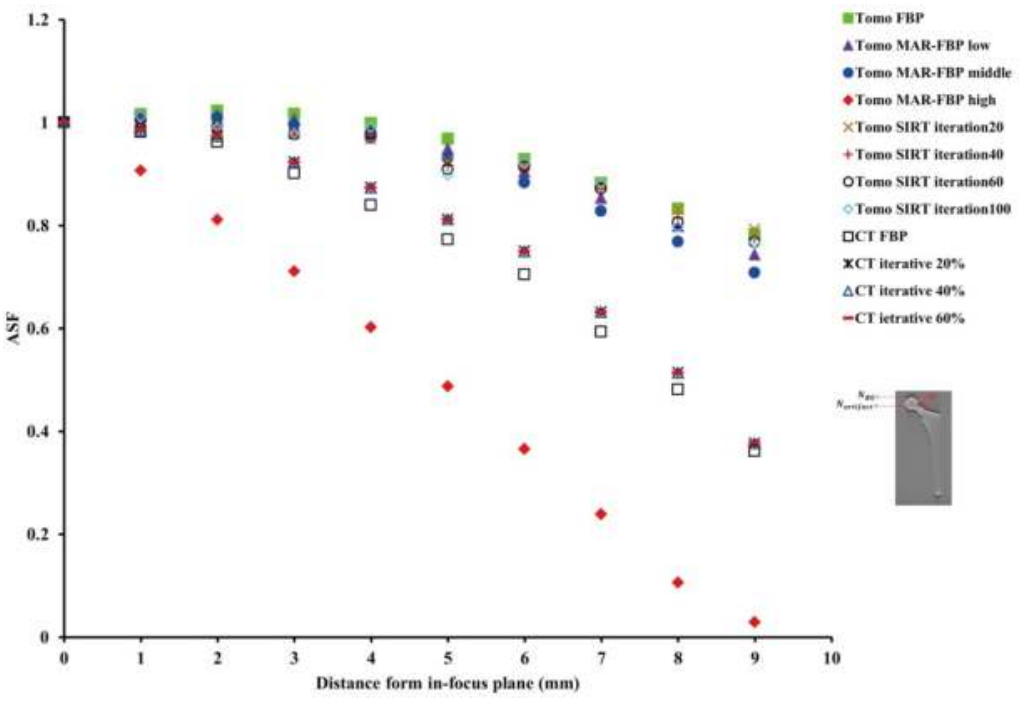

Figure 7. Comparison between ASF versus distance from the in-focus plane for tomosynthesis and CT. ASF chart demonstrates that tomosynthesis with metal-artifact-reduction processing results in the maximum removal of metal artifacts.

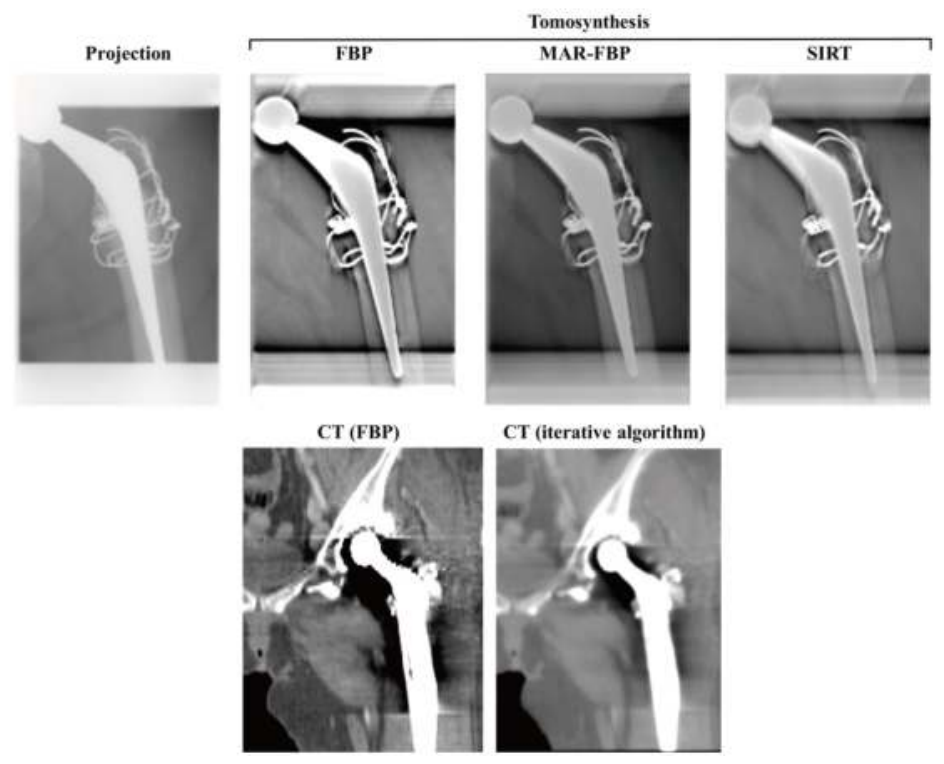

Figure 8. Case patient (52-year-old woman; coxarthrosis, after total hip arthroplasty). The use of metal-artifact-reduction processing tomosynthesis allowed a better visualisation of the left hip joint prosthesis by blurring the anatomic structures above and below the visualized planes. (MAR; metal-artifact-reduction) 


\section{Conclusion}

Applicaton of metal-artifact-reduction processing digital tomosynthesis in imaging prostheses appears promising. The results of a prosthesis study suggest that digital tomosynthesis (metal-artifact-reduction processing and an iterative reconstruction algorithm) can produce improved image quality compared with that by conventional FBP tomosynthesis by removing the overlying structures and providing limited 3D information. In addition, the digital tomosynthesis method apparently facilitates the significant improvement of images corrupted by metal artifacts. Metal-artifact-reduction processing digital tomosynthesis provided higher quality images compared to those by CT. Metal-artifact-reduction processing digital tomosynthesis is the best solution when the high-attenuation feature causing the artifacts can be segmented accurately from the projection.

On the whole, metal-artifact-reduction processing performed satisfactorily, but its effectiveness depended strongly on the image region. Metal-artifact-reduction processing digital tomosynthesis images yielded good results, which were independent of the type of metal present in the phantom study or patient, and showed good removal of metal artifacts, particularly at greater distances from the metal objects. Flexibility in selecting the imaging parameters in metal-artifact-reduction processing digital tomosynthesis on the basis of the desired final images and realistic imaging conditions may be beneficial.

\section{Author details}

Tsutomu Gomi ${ }^{1}$, Hiroshi Hirano ${ }^{2}$ and Masahiro Nakajima ${ }^{3}$

1 School of Allied Health Sciences, Kitasato University, Japan

2 Department of Radiology, Shinshu University Hospital, Japan

3 Department of Radiology, Dokkyo Medical University Hospital, Japan

\section{References}

[1] Gordon R, Bender R, Hermen GT. (1970) Algebraic reconstruction techniques (ART) for three-dimensional electron microscopy and x-ray photography. Journal of theoretical biology, 29, pp.471-481 ISSN 0022-5193

[2] Ziedses des, Plante BG. (1932) Eine neue methode zur differenzierung in der roentgenographie (planigraphie). Acta radiologica, 13, pp.182-92, ISSN 0284-1851

[3] Miller ER, McCurry EM, Hruska B. (1971) An infinite number of laminagrams from a finite number of radiographs. Radiology, 98, pp.249-255, ISSN 0033-8419 
[4] Grant DG. Tomosynthesis. (1972) A three-dimensional radiographic imaging technique. IEEE transactions on bio-medical engineering, 19, pp.20-8, ISSN 0018-9294

[5] Baily NA, Lasser EC, Crepeau RL. (1973) Electrofluoro-plangigraphy. Radiology, 107, pp.669-671 ISSN 0033-8419

[6] Kruger RA, Nelson JA, Ghosh-Roy et al. (1983) Dynamic tomographic digital subtraction angiography using temporal filteration. Radiology, pp.863-867, ISSN 0033-8419

[7] Sone S, Kasuga T, Sakai S et al. (1991) Development of a high-resolution digital tomosynthesis system and its clinical application. Radiographics, 11, pp.807-822, ISSN 0271-5333

[8] Stiel G, Stiel LG, Klotz E et al. (1993) Digital flashing tomosynthesis: A promising technique for angiographic screening. IEEE transactions on medical imaging, 12, pp. 314-21, ISSN 0278-0062

[9] Duryea J, Dobbins JT, Lynch JA. (2003) Digital tomosynthesis of hand joints for arthritis assessment. Medical Physics, 30, pp.325-33, ISSN 0094-2405

[10] Sone S, Kasuga T, Sakai F, et al. (1995) Image processing in the digital tomosynthesis for pulmonary imaging. European Radiology, 5, pp.96-101, ISSN 0938-7994

[11] Niklason LT, Christian BT, Niklason LE, et al. (1997) Digital tomosynthesis in breast imaging. Radiology, 205, pp.399-406, ISSN 0033-8419

[12] Dobbins JT III, Godfrey DJ. (2003) Digital x-ray tomosynthesis: curent state of the art and clinical potential. Physics in medicine and biology, 48, R65-106, ISSN 0031-9155

[13] Kak A, Slaney M. (1988) Principles of computerized tomographic imaging. IEEE, ISBN 0-89874-494-X, New York

[14] Smith DB. (1985) Image reconstruction from cone-beam projections: necessary and sufficient conditions and reconstruction methods. IEEE transactions on medical imaging, M1-4, pp.14-25, ISSN 0278-0062

[15] Feldkamp LA, Davis LC, Kress JW. (1984) Practical cone-beam algorithm. Journal of the Optical Society of America, A1, pp.612-619, ISSN 0030-3941

[16] Ruttimann U, Groenhuis R, Webber R. (1984) Restoration of digital multilane tomosynthesis by a constrained iteration method. IEEE transactions on medical imaging, MI-3, pp.141-8, ISSN 0278-0062

[17] Bleuet P, Guillemaud R, Magin I. Et al. (2002) An adapted fan volume sampling scheme for 3D algebraic reconstruction in linear tomosynthesis. IEEE transactions on nuclear science, 49, pp.2366-72, ISSN 0018-9499

[18] Hsieh J. (1995) computed tomography technology and applications; Image artifacts causes and correction, In: Medical CT and ultrasound, LW Goleman, JB Fowlkes, pp. 487-518, Advanced Medical Publishing ISBN 1-883526-03-5, Madison 
[19] Kamel EM, Burger C, Buck A, et al. (2003) Impact of metallic dental implants on CTbased attenuation correction in a combined PET/CT scanner. European Radiology, 13, pp.724-8, ISSN 0938-7994

[20] Wang G, Snyder DL, O'Sullivan JA, et al. (1996) Iterative debluring for metal artifacts reduction. IEEE transactions on medical imaging, 15, pp.657-664, ISSN 0278-0062

[21] Wang G, Vannier MW, Cheng PC. (1999) X-ray cone-beam tomography for metal artifacts reduction and local region reconstruction. Microscopy and microanalysis, 5, pp.58-65, ISSN 1431-9276

[22] Wang G, Frei T, Vannier MW. (2000) A fast iterative algorithm for metal artifact reduction in x-ray CT. Academic Radiology, 7, pp.607-614, ISSN 1076-6332

[23] Man B De, Nuyts J, Dupont P, et al. (2000) Reduction of metal streak artifacts in x-ray computed tomography using a transmission maximum a posteriori algorithm. IEEE transactions on nuclear science, 47, pp.997-981, ISSN 0018-9499

[24] Robertson DD, Yuan J, Wang G, et al. (1997) Total hip prosthesis metal-artifact suppression using iterative deblurring reconstruction. Journal of computer assisted tomography, 21, pp.293-298, ISSN 0363-8715

[25] Wu T, Stewart A, Stanton M, McCauley T, et al. (2003) Tomographic mammography using a limited number of low-dose cone-beam projection images. Medical Physics, 30, pp.265-380, ISSN 0094-2405

[26] Gomi T, Hirano H. (2008) Clinical potential of digital linear tomosynthesis imaging of total joint arthroplasty. Journal of Digital Imaging, 21, pp.312-22, ISSN 0897-1889

[27] Gomi T, Hirano H, Umeda T. (2009) Evaluation of the X-ray digital linear tomosynthesis reconstruction processing method for metal artifact reduction. Computerized Medical Imaging and Graphics, 33, pp.257-274, ISSN 0895-6111

[28] Hara AK, Paden RG, Silva AC et al. (2009) Iterative reconstruction technique for reducing body radiation dose at CT. American Journal of Roentgenology, 193, pp. 764-771, ISSN 0361-803X

[29] Marin D, Nelson RC, Schindera ST et al. (2010) Low-tube-voltage, high-tube-current multidetector abdominal CT: improved image quality and decreased radiation dose with adaptive statistical iterative reconstruction algorithm-initial clinical experience. Radiology, 254, pp.145-153, ISSN 0033-8419

[30] Yanagawa M, Honda O, Yoshida S et al. (2010) Adaptive statistical iterative reconstruction technique for pulmonary CT: image quality of the cadaveric lung on standard- and reduced-dose CT. Academic Radiology, 17, pp.1259-1266, ISSN 1076-6332

[31] Gomi T, Hirano H, Nakajima M. (2012) Recent advance in arthroplasty. "Chapter 6 xray digital linear tomosynthesis imaging of arhtoroplasty", pp.95-108, INTECH, ISSN 978-953-307-990-5, Croatia 
